\title{
Osteogenic Differentiation of Mesenchymal Stem Cells in their Native Niche Like Environment
}

Fakhr E $\mathrm{E}^{1}$, Seyedjafari $\mathrm{E}^{2}$, Ahmadbeigi $\mathrm{N}^{3 *}$

${ }^{1}$ SABZ Biomedical Science-Based Company, Tehran, Iran.

${ }^{2}$ Department of Biotechnology, College of Science, University of Tehran, Tehran, Iran.

${ }^{3}$ Liver and pancreatobiliary diseases research center, Digestive Disease Research Institute, Tehran. University of Medical Sciences, Tehran, Iran.

\section{Abstract}

Niche is an important microenvironment regulating stem cell's natural behavior and its fate. Mesenchymal Stem Cells (MSCs) are the most common cells used in bone tissue engineering. However, common culture procedures do not retain MSCs in their niche and apply in vitro expansion which can cause abnormal behavior. In this study, we propose that, by Keeping MSCs in their native niche, natural behavior of these cells and their interactions are maintained and the quality of differentiation will enhance. In our previous studies, we isolated MSCs kept in their native niche-like environment (nativeMSCs) in the form of aggregates. Herein, in order to explore the osteogenic differentiation of these cells, they were isolated from bone marrow, and in vitro osteogenesis was assessed via alizarin red and real time PCR. It was observed that they differentiated after 6 days, which is remarkably faster than the conventional 21-day period. Higher expression of Runx2, Spp1 and alkaline phosphatase in native-MSCs also confirmed in vitro results. We also examine their attachment on poly (L-lactic acid) (PLLA) scaffold. In sum, native-MSCs exhibited faster differentiation than expanded MSCs which proves the importance of keeping stem cells in their niche.

Keywords: Niche; Messenchymal Stem Cell; Osteogenic Differentiation; Bone Tissue Engineering.

List of Abbreviation: Native-MSCs: MSCs which are kept in their niche like environment;

Ex-MSCs: Expanded MSCs are common MSCs which isolate and expand based on their adherent properties.

\section{*Corresponding Author:}

Naser Ahmadbeigi PhD,

Tehran University of Medical Sciences, Digestive Disease Research Institute, Shariati Hospital, North Kargar Ave.,Tehran, 14117, Iran.

Tel: $+98-21-88003140$

Fax: +98-21-88634118

Email: N-Ahmadbeigi@tums.ac.ir

Received: July 27, 2015

Accepted: December 07, 2015

Published: December 10, 2015

Citation: Fakhr E, Seyedjafari E, Ahmadbeigi N (2015) Osteogenic Differentiation of Mesenchymal Stem Cells in their Native Niche Like Environment. Int J Stem Cell Res Transplant 03(6), 129-133. doi: http:// dx.doi.org/10.19070/2328-3548-1500022

Copyright: Ahmadbeigi $\mathbf{N}^{\circ}$ 2015. This is an open-access article distributed under the terms of the Creative Commons Attribution License, which permits unrestricted use, distribution and reproduction in any medium, provided the original author and source are credited.

\section{Introduction}

Niche, as an important microenvironment first proposed by R.Schofield in 1978 [1], is responsible for regulating and preserving natural behavior of stem cells. Any stem cell's niche substantially consists of the stem cell, mesenchymal or stromal cells, cell-cell, cell-extracellular matrix interactions, and external signals from other sources through blood vessels and neurons. These intrinsic and extrinsic factors influence the behavior and fate of stem cells as well as the shape, differentiation, and proliferation of them $[2,3]$. The importance of niche is obvious as Leanne Jones (2008) has entitled it as a home for stem cells [4].

Mesenchymal Stem Cells (MSCs) have attracted great attention in regenerative medicine because of their multilineage potential and easy isolation from bone marrow, adipose tissue, and the umbilical cord $[5,6]$. It is shown that bone marrow-derived MSCs (BmMSCs) are the most common cells used in bone tissue engineering $[7,8]$. However, lack of identical markers for their selection and their low quantity leads to usage of cell culture procedures which are mainly based on plastic adhesion property and do not retain MSCs in their native niche. This can lead to detrimental consequences and affect MSCs natural behaviour, so that raise concerns about their safety for cell-based treatments [9-11]. In our previous studies, in accordance with other studies, we explain how in vitro culture of MSCs cause the chromosomal abnormality of these cells, which may influence vital properties of them such as homing ability $[12,13]$. Due to unpredictable time of bone surgery and its urgency, using autologous MSCs seems to be farfetched since for having a considerable amount of cells necessary for bone regeneration a minimum of a two-week culture is needed [14].

Some studies have attempted to use uncultured mononuclear cells (Bm-MNCs) as a source of MSCs in order to prevent the in vitro culture of MSCs and have used them more instantly [15, 16]. Although $\mathrm{Bm}-\mathrm{MNCs}$ have recently gained a great interest for being applied in stem cell therapies, the quantity of MSCs in Bm-MNCs are very low [17]. For this aim, a suitable source of cells contain- 
ing sufficient MSCs kept in their niche, without the need of in vitro expansion, is favored.

In our previous study, we suggested that MSCs have aggregate nature and ex vivo expansion of MSCs may become unnecessary because of having adequate multipotent cells [18]. By omitting in vitro expansion, intrinsic properties of MSCs will be sustained and can be used instantly for urgent situations. We also showed that these cell complexes had niche-like properties and, using this protocol, MSCs can be kept in their native niche-like environment [19]. In this study, we mainly focus on osteogenic differentiation of MSCs in their niche-like environment derived from BM, referred to as native-MSCs. We also investigate the feasibility of their attachment to scaffold for bone tissue engineering purposes.

\section{Material and Methods}

Isolation of mesenchymal stem cells in their niche-like environment (native-MSCs) and expanded mesenchymal stem cells (ex-MSCs)

C57BL/ 6 mice were used as the source of native-MSCs and exMSCs. The mice euthanized morally and the ends of the tibia and femur of both legs were cut to extract the bone marrow in a centrifuge tube being centrifuged for $1 \mathrm{~min}$ at $800 \mathrm{~g}$. Bone marrow samples were diluted with phosphate-buffered saline plus the Ethylenediamine tetra acetic acid (PBS/EDTA; GIBCO-BRL, GrandIsland, NY, USA) and filtered through a c. 20- $\mu \mathrm{m}$ strainer (a double-layered 40- $\mu \mathrm{m}$ nylon mesh; BD Biosciences, San Jose, CA, USA). Filter-retained fraction includes MSCs divided in two parts. One part was used as a source of native-MSCs without in vitro expansion, and another part was cultured with common procedure based on adherent properties to culture ex-MSCs, as described previously [12].

\section{Osteogenic differentiation and alizarin red staining}

In order to explore osteogenic differentiation, native-MSCs, instantly after isolation, and ex-MSCs were treated with Dulbecco 's modified Eagle's medium (DMEM) containing 10\% fetal bovine serum (FBS) (both from GIBCO-BRL) plus $10 \mathrm{mM}$ beta-glycerol phosphate (Merck, Darmstadt, Germany), $50 \mu \mathrm{g} / \mathrm{mL}$ ascorbic acid bi-phosphate (Sigma, St Louis, MO, USA), and $100 \mathrm{nM}$ dexamethasone (Sigma). The medium was changed every 2 days.

For confirming in vitro osteogenic differentiation of native-MSCs in comparison with ex-MSCs, alizarin red staining was performed. The cells were fixed with formaldehyde $4 \%$ for $10 \mathrm{~min}$ at room temperature, washed with PBS twice, and stained with $2 \%$ alizarin red solution $(\mathrm{pH}=4)$ for $15 \mathrm{~min}$ at room temperature. The results were assessed by alizarin red staining and compared on days 6,11 , and 21 , respectively.

\section{Real time RT- PCR}

The expression of some osteogenic genes in native-MSCs and exMSCs was evaluated. Total RNA of cells on day 1, 3, and 6 was extracted and random hexamer primed cDNA synthesis was performed by Takara cDNA synthesis kit (Japan, clontec). Real-time PCR was done via MaximaTM SYBR Green/ROX qPCR Master Mix (Fermentas). 40 cycles PCR was performed in Applied Bio systems $^{\circledR} 7500$ Real-Time PCR Systems (life technologies, UK) and the melting curves were analyzed for assessing PCR specificity. All the reactions were repeated twice and the averages of threshold cycles were considered. REST software was used for data analysis. $\Delta \Delta \mathrm{Ct}$ method was used for comparing relative expression and Beta-actin was used as a housekeeping gene for normalization. Related primers are shown in Table 1.

\section{Fabrication of poly (L-lactic acid) scaffolds}

This kind of scaffold was prepared by electrospinning technique; $0.45 \mathrm{~g}$ of poly (L-lactic acid) (PLLA) (sigma) was diluted in $9 \mathrm{ml}$ chloroform (Merck, Germany) and $1 \mathrm{ml}$ Dimethylformamide to reach $4.5 \%(\mathrm{w} / \mathrm{v})$ ratio. The solution was poured in a $10 \mathrm{ml}$ syringe with a 21 -guage needle. A rotational collector, placed in a 15-cm distance from the needle, was used for collecting the electrospun fibers. The flow rate was set to $0.5 \mathrm{ml} / \mathrm{h}$, and $10 \mathrm{Kv}$ voltage was used for transferring the solution droplet from the needle to the collector. After gaining $200 \mu \mathrm{m}$ thickness, the mat was separated from the collector and the residual solvent was evaporated by vacuuming.

\section{Cell seeding}

Native-MSCs were seeded on PLLA scaffolds and their attachment was examined for tissue engineering purposes. Before cell seeding, PLLA scaffolds were cut into a $2-\mathrm{cm}$ diameter circular shape and both sides of them were sterilized with UV for 20 minutes. Due to the aggregate nature of our cell complexes, we were not able to count the cells. Therefore, $200 \mu$ of filter retained fraction including native-MSCs was seeded on scaffolds, and basal medium was added to reach the final volume of $1 \mathrm{ml}$.

\section{Scanning electron microscopy (SEM)}

The surface morphology of scaffolds was assessed via a scanning electron microscope. The attachments of native-MSCs were also determined by scanning electron microscopy. Also, DAPI test was done in evaluating the presence of cells on the scaffolds before performing SEM.

\section{Statistical analysis}

All the experiments were repeated twice. The data are shown as mean \pm standard deviation. The statistics were conducted via

Table 1. Sequence of primers for quantitative real-time PCR.

\begin{tabular}{|c|c|c|}
\hline genes & Forward primer & Reverse primer \\
\hline Runx 2 & 5'-AATGCCTCCGCTGTTATG-3' & 5'-TCTGTCTGTGCCTTCTTGG-3' \\
\hline Spp1 & 5'-AACCAGCCAAGGACTAACTAC-3' & 5'-CTTCAGAGGACACAGCATTC-3' \\
\hline Alkaline phosphatase & 5'-GGTAGAT'TACGCTCACAACAAC-3' & 5'-CAGGCACAGTGGTCAAGG-3' \\
\hline Beta-actin & 5'-CT'TCTTGGGTATGGAATCCTG -3' & 5'-GTGTTGGCATAGAGGTCTTTAC-3' \\
\hline
\end{tabular}


IBM SPSS statistics developer 20.0 and statistical significance was set at $\mathrm{p}<0.05$.

\section{Results}

Native-MSCs showed faster osteogenic differentiation than ex-MSCs

Ex-MSCs and native-MSCs were harvested and characterized as described before, and were treated with osteogenic medium [11]. However, native-MSCs were instantly treated by differentiation medium. Osteogenic outcomes of native-MSCs and ex-MSCs revealed a meaningful difference compared to their controls, indicating a proper differentiation induced by osteogenic medium. Native-MSCs showed osteogenesis on day 6, but no differentiation was screened for ex-MSCs on the respective day. Osteogenesis of native-MSCs increased on day 11 and ex-MSCs, still, had no osteogenic differentiation. By completing the 21-day interval, both native-MSCs and ex-MSCs had Osteogenesis. Control of native-MSCs showed no differentiation even after passing 21 days (Figure 1).

Bone-related genes expressed higher in native-MSCs on early days

As osteogenesis of native-MSCs was seen on day 6, we proposed that alteration in the expression pattern of osteogenic genes may initiate before the $6^{\text {th }}$ day; hence, time points prior to this time, including day 6, was explored. Three osteogenic genes were compared on day 1, 3, and 6 in ex-MSCS and native-MSCs after differentiation. The expressions of Runx2 and Spp1 in native-MSCs and ex-MSCS had an ascendant trend. Their expressions were significantly higher in native-MSCs compared to ex-MSCs. NativeMSCs showed a higher level of Spp1 than ex-MSCs on the first day. Although the difference between the expression of $s p p 1$ in native-MSCs and ex-MSCs was decreased on day 3 and 6, Spp1 was expressed higher in native-MSCs. While an increase in alkaline phosphatase expression level was detected on the first day of osteogenic differentiation of native-MSCs, a decrease was observed in the succeeding days. By the $6^{\text {th }}$ day, the expression of alkaline phosphatase was higher in ex-MSCS. Runx2 was expressed more in native-MSCs than ex-MSCs on all respective days and the level of this transcript was remarkably higher in native-MSCs compared to ex-MSCs on day 6 (Figure2).

\section{Native-MSCs attached properly on PLLA scaffold}

Based on SEM photos, PLLA scaffold benefitted appropriate fibrous structure and the native-MSCs were attached and distributed on the scaffold properly. SEM photos also showed the aggregate nature of our derivative cell complexes (Figure3).

\section{Discussion}

In this study, the osteogenesis of MSCs kept in their native nichelike environment (native-MSCs) was investigated. These cells were derived from bone marrow and showed efficient and faster osteogenic differentiation.

We observed that native-MSCs differentiated on day 6, which is significantly shorter than the conventional 21-day period for osteogenic differentiation. Although native-MSCs's osteogenesis increased on day 11, ex-MSCs showed no osteogenesis. We suppose this notable potential to be related to using native-MSCs instantly; so, the repercussions of ex vivo expansion were omitted. Without in vitro expansion, native phenotype and genetic stability of MSCs will not be interrupted so they can respond to stimuli of differentiation faster than ex-MSCs [20, 21]. Real-time results also confirmed this remarkable osteogenic potential.

On days 1, 3, and 6, Runx2 was expressed higher in native-MSCs than ex-MSCs. High levels of this gene, as an essential transcription factor in osteogenesis causing osteoblast differentiation, resulted in osteogenesis [22]. Our results confirmed the correlation whereby high level of Runx2 leads to increase in Spp1 [23]. Spp1 is the murine ortholog of Osteopontin, and is one of the extracellu-

Figure 1. Native-MSCs showed osteogenesis on day 6 and their osteogenic differentiation increased remarkably on day 11 and 21, respectively. However, ex-MSCs showed no osteogenesis on day 6 and 11. Osteogenesis of ex-MSCs was detectable on day 21. All of the controls of native-MSCs and ex-MSCs showed no differentiation on respective days. Scale bars: $100 \mu \mathrm{m}$.

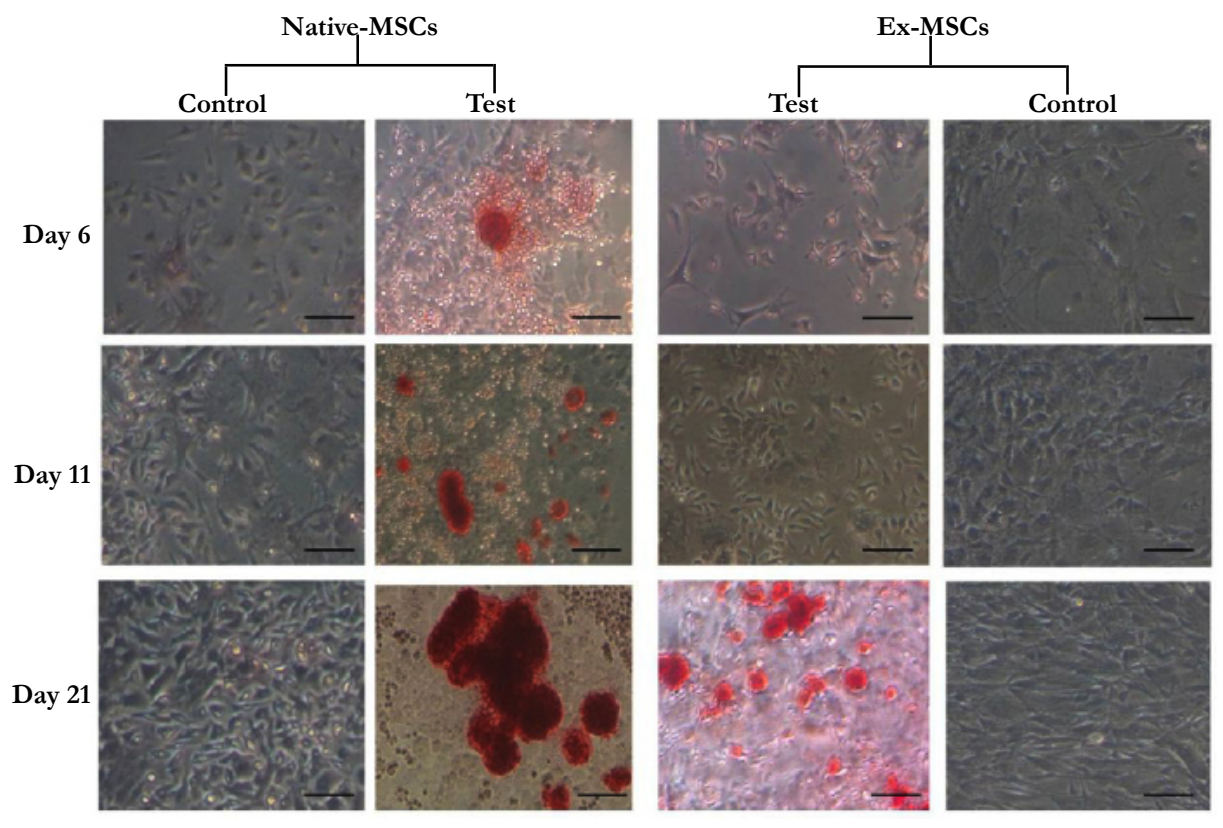


Figure 2. Expression of bone related genes in native-MSCs and ex-MSCs on days 1, 3 and 6. Standard deviations are shown by bars and asterisk shows significant difference with $\mathrm{p}<0.05$.
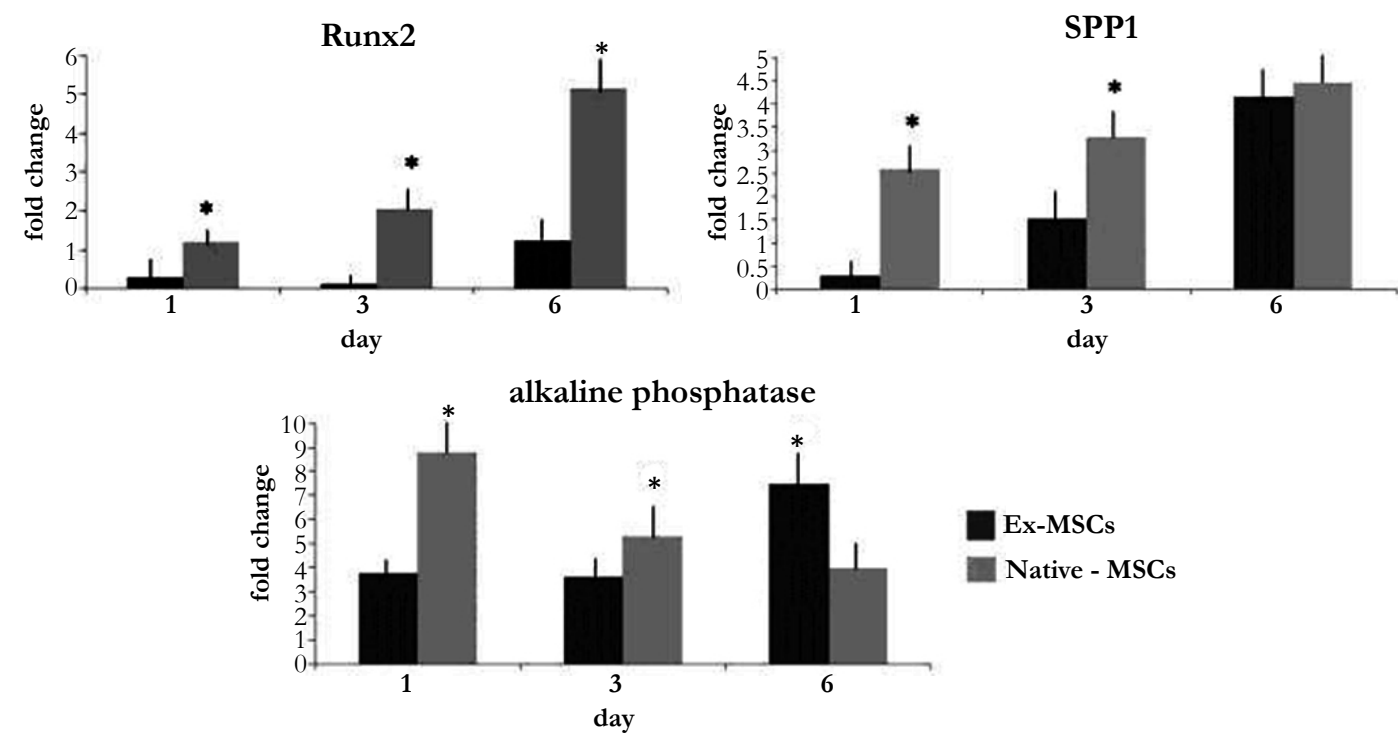

Figure 3. Morphology of PLLA scaffolds (A), native-MSCs attachment on PLLA scaffold (B).
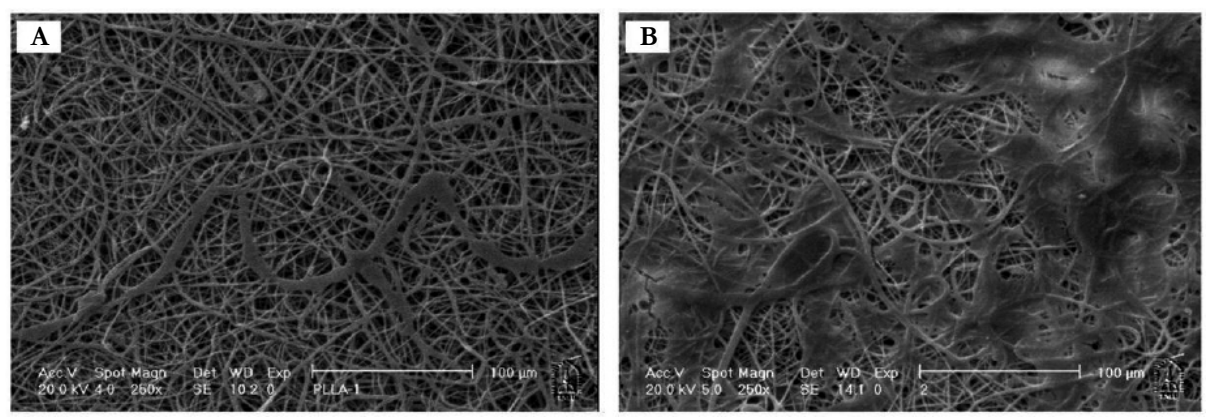

lar bone proteins which mainly induces natural mineralization and bone formation and is expressed by a variety of cells, including osteoblasts and osteocytes [24, 25]. Higher expression of alkaline phosphatase on early days in native-MSCs is another clue for fast bone regeneration. Previously, in accordance with our findings, McNamara LM. et al (2012) illustrated that culture of MSCs with conditioned medium derived from osteocytes caused an increase in alkaline phosphatase level on the early days and a decrease as the cells matured and mineral deposition happened. While in their study attempts were made to mimic niche-like environment for faster differentiation of MSCs [26], we introduced a cell source in which MSCs are kept in their natural niche like environment without any manipulation.

As our technique used native-MSCs instantly, it can be a suitable source for bone tissue engineering. Therefore, we examined the attachment of these cells on PLLA scaffolds. This scaffold is a promising candidate for tissue engineering purposes because of its integrity and supporting cell proliferation [27]. It was observed that native-MSCs were attached and distributed properly on this scaffold.

Mimicking and manipulating native niche has been explored recently in order to improve bone tissue engineering and also studying the niche mediated regulation of MSCs [28-30].

Using MSCs in their own niche derived from bone marrow can be worthwhile because they are kept in a native microenvironment with all intrinsic cellular interactions and signaling. By not separating MSCs from their native niche, lots of cell-cell, ECM and cytoskeleton interactions, and cell signaling which are pivotal for the natural behavior of MSCs are maintained [31, 32]. Our results found this protocol promising due to the fact that MSCs were not expanded in vitro and were kept in their niche-like environment so that the intrinsic behaviors of stem cells were retained. This study indicates notable osteogenic properties of native-MSCs and, for the first time, makes it possible to differentiate MSCs in their native niche-like environment. Because of their independence from in vitro expansion, they can be also suggested as a beneficial source for instant cell therapy. While further studies are needed to identify the distinct component of these niche-like environments, it is also worthwhile to examine the multilineage differentiation of native-MSCs and their usage in bone tissue engineering and other subclasses of regenerative medicine.

\section{Acknowledgments}

This study was supported by a grant from SABZ Biomedical Science-Based Company, Tehran, Iran.

\section{References}

[1]. Schofield R (1978) The relationship between the spleen colonyforming cell and the haemopoietic stem cell. Blood cells 4(1-2): 7-25.

[2]. Tran E, Chinnasamy D, Yu Z, Morgan RA, Lee CC, et al. (2013) Immune targeting of fibroblast activation protein triggers recognition of multipotent bone marrow stromal cells and cachexia. J Exp Med 210(6): 1125-1135.

[3]. Noll JE, Williams SA, Purton LE, Zannettino ACW (2012) Tug of war in the haematopoietic stem cell niche: do myeloma plasma cells compete for the HSC niche?. Blood Cancer Journal 2(9): e91.

[4]. Jones DL, Wagers AJ (2008) No place like home: anatomy and function of 
the stem cell niche. Nat Rev Mol Cell Biol 9(1): 11-21.

[5]. Pittenger MF, Mackay AM, Beck SC, Jaiswal RK, Douglas R, et al. (1999) Multilineage potential of adult human mesenchymal stem cells. Science 284(5411): 143-147.

[6]. Hass R, Kasper C, Bohm S, Jacobs R (2011) Different populations and sources of human mesenchymal stem cells (MSC): a comparison of adult and neonatal tissue-derived MSC. Cell Commun Signal 9: 12.

[7]. Shafiee A, Seyedjafari E, Soleimani M, Ahmadbeigi N, Dinarvand P, et al. (2011) A comparison between osteogenic differentiation of human unrestricted somatic stem cells and mesenchymal stem cells from bone marrow and adipose tissue. Biotechnol Lett 33(6): 1257-1264.

[8]. Im GI, Shin YW, Lee KB (2005) Do adipose tissue-derived mesenchymal stem cells have the same osteogenic and chondrogenic potential as bone marrow-derived cells? Osteoarthritis Cartilage 13(10): 845-853.

[9]. Soleimani M, Nadri S (2009) A protocol for isolation and culture of mesenchymal stem cells from mouse bone marrow. Nat Protoc 4(1): 102-106.

[10]. Prockop DJ, Olson SD (2007) Clinical trials with adult stem/progenitor cells for tissue repair: let's not overlook some essential precautions. Blood 109(8): 3147-3151.

[11]. Nauta AJ, Fibbe WE (2007) Immunomodulatory properties of mesenchymal stromal cells. Blood 110(10): 3499-3506.

[12]. Ahmadbeigi N, Soleimani M, Gheisari Y, Vasei M, Amanpour S, et al. (2011) Dormant phase and multinuclear cells: two key phenomena in early culture of murine bone marrow mesenchymal stem cells. Stem Cells Dev 20(8): 1337-1347.

[13]. Foudah D, Redaelli S, Donzelli E, Bentivegna A, Miloso M, et al. (2009) Monitoring the genomic stability of in vitro cultured rat bone-marrow-derived mesenchymal stem cells. Chromosome Res 17(8): 1025-1039.

[14]. Pérez-Simon JA, Lopez-Villar O, Andreu EJ, Rifon J, Muntion S, et al. (2011) Mesenchymal stem cells expanded in vitro with human serum for the treatment of acute and chronic graft- versus-host disease: results of a phase I/ II clinical trial. Haematologica 96(7): 1072-1076.

[15]. Kretlow JD, Spicer PP, Jansen JA, Vacanti CA, Kasper FK, et al. (2010) Uncultured marrow mononuclear cells delivered within fibrin glue hydrogels to porous scaffolds enhance bone regeneration within critical-sized rat cranial defects. Tissue Eng Part A 16(12): 3555-3568.

[16]. Chang F, Ishii T, Yanai T, Mishima H, Akaogi H, et al. (2008) Repair of large full- thickness articular cartilage defects by transplantation of autologous uncultured bone- marrow-derived mononuclear cells. J Orthop Res 26(1): 18-26.

[17]. Alvarez-Viejo M, Menendez-Menendez Y, Blanco-Gelaz M, Ferrero-Gutierrez A, Fernandez-Rodriguez M, et al. (2013) Quantifying mesenchymal stem cells in the mononuclear cell fraction of bone marrow samples obtained for cell therapy. Transplantation Proc 45(1): 434-439.

[18]. Ahmadbeigi N, Soleimani M, Babaeijandaghi F, Mortazavi Y, Gheisari
Y, et al. (2012) The aggregate nature of human mesenchymal stromal cells in native bone marrow. Cytotherapy 14(8): 917-924.

[19]. Ahmadbeigi N, Soleimani M, Vasei M, Gheisari Y, Mortazavi Y, et al. (2013) Isolation, characterization, and transplantation of bone marrow-derived cell components with hematopoietic stem cell niche properties. Stem Cells Dev 22(23): 3052-3061.

[20]. Agata H, Asahina I, Watanabe N, Ishii Y, Kubo N, Ohshima S, et al. (2010) Characteristic change and loss of in vivo osteogenic abilities of human bone marrow stromal cells during passage. Tissue Eng Part A 16(2): 663-673.

[21]. Amiri F, Jahanian-Najafabadi A, Roudkenar MH (2015) In vitro augmentation of mesenchymal stem cells viability in stressful microenvironments : In vitro augmentation of mesenchymal stem cells viability. Cell Stress Chaperones 20(2): 237-251.

[22]. Shui C, Spelsberg TC, Riggs BL, Khosla S (2003) Changes in Runx2/Cbfa1 expression and activity during osteoblastic differentiation of human bone marrow stromal cells. J Bone Miner Res 18(2): 213-221.

[23]. Nakashima K, Zhou X, Kunkel G, Zhang Z, Deng JM, et al. (2002) The novel zinc finger-containing transcription factor osterix is required for osteoblast differentiation and bone formation. Cell 108(1): 17-29.

[24]. Choi ST, Kim JH, Kang EJ, Lee SW, Park MC, et al. (2008) Osteopontin might be involved in bone remodelling rather than in inflammation in ankylosing spondylitis. Rheumatology 47(12): 1775-1779.

[25]. McKee MD, Addison WN, Kaartinen MT (2005) Hierarchies of extracellular matrix and mineral organization in bone of the craniofacial complex and skeleton. Cells Tissues Organs 181(3-4): 176-188.

[26]. Birmingham E, Niebur GL, McHugh PE, Shaw G, Barry FP, et al. (2012) Osteogenic differentiation of mesenchymal stem cells is regulated by osteocyte and osteoblast cells in a simplified bone niche. Eur Cell Mater 23: 1327.

[27]. Li WJ, Cooper JA, Mauck RL, Tuan RS (2006) Fabrication and characterization of six electrospun poly ( $\alpha$-hydroxy ester)-based fibrous scaffolds for tissue engineering applications. Acta Biomater 2(4): 377-385.

[28]. Lane SW, Williams DA, Watt FM (2014) Modulating the stem cell niche for tissue regeneration. Nat Biotechnol 32(8): 795-803.

[29]. Lu ZF, Wang G, Zreiqat H (2014) Engineering Bone Niche Signals to Control Stem Cell Fate for Bone Tissue Regeneration. Arch Stem Cell 1(1): 1003.

[30]. Sharma MB, Limaye LS, Kale VP (2012) Mimicking the functional hematopoietic stem cell niche in vitro: recapitulation of marrow physiology by hydrogel-based three-dimensional cultures of mesenchymal stromal cells. Haematologica 97(5): 651-660.

[31]. Celso CL, Scadden DT (2011) The haematopoietic stem cell niche at a glance. J Cell Sci 124(Pt 21): 3529-3535.

[32]. Purton LE, Scadden DT (2008) The Hematopoietic Stem Cell Niche. 